Assiut Scientific Nursing Journal

http://asnj.journals.ekb.eg

http://www.arabimpactfactor.com

\title{
Self-Care BehaviorFor Hemodialysis Patients At Beni-Suef University Hospital
}

\author{
Omar Fathy Hamza ${ }^{1}$, Safaa Ahmed Mohamed ${ }^{2}$, Rabaa Hamed Hassanen ${ }^{3}$ \& Abeer Mohammed Elmaghawry ${ }^{4}$ \\ 1. Assistant lecturer in family and Community Health Nursing- Faculty of Nursing- Beni Suef University, Egypt. \\ 2. Professor of Community Health Nursing, Faculty of Nursing, Assiut University, Egypt. \\ 3. Professor of Community Health Nursing, Faculty of Nursing, Assiut University, Egypt. \\ 4. Assistant Professor of Community Health Nursing, Faculty of Nursing, Damietta University, Egypt.
}

\begin{abstract}
:
Kidney diseases influence over 750 million persons all over the world. Chronic kidney disease is a progressive disease requires hemodialysis or other treatments to prevent death in the late stages. Aim: To assess self-care behavior for hemodialysis patients in dialysis unit. Subjects \& methods: A descriptive research design has been used in this study. It was conducted at BeniSuef University hospital. A 55 patient who attend the dialysis unit at Beni-Suef university hospital and meet the inclusion criteria was participated in this study. Three tools were used:

Tools: The first tool: A structured interviewing questionnaire that include two parts; Part 1: socio-demographic characteristics of hemodialysis patients. Part 2: Past history of hemodialysis patients. Second tool: A knowledge assessment data sheet of hemodialysis patients used to assess knowledge of hemodialysis patients. Third tool: Selfcare measurement scale used to measure practice regarding health behavior of hemodialysis patients. Results: The mean age of the participants was $38.61 \pm 6.32$. 34.6\% of them were illiterate. $80 \%$ of them had poor knowledge and all patients had poor practice regarding health behavior. Conclusion: The current study concluded that the majority of them had had poor knowledge level and all patients had poor practice regarding health behavior. Recommendations: Design instruction model for nurses to follow up periodically with hemodialysis patients about practicing self-care behavior.
\end{abstract}

\section{Keywords: Hemodialysis Patients. Self-Care Behavior \& Dialysis Unit.}

\section{Introduction}

The kidneys regulate body fluids, electrolytes, and acid-base balance, among other things (Qian, 2017). Over 750 million people worldwide are affected by kidney disease (Crews, et al., 2018).

Chronic renal disease is a progressive condition that necessitates hemodialysis or other therapies in its latter stages to avoid death. (Rini, et al., 2021). Reduced renal function, as evidenced by a glomerular filtration rate of less than $60 \mathrm{ml} / \mathrm{min} / 1.73 \mathrm{~m}^{2}$ and/or indicators of renal impairment lasting three months or longer, characterizes chronic kidney disease. (Webster et al., 2017).

Globally, an estimated 850 million people are affected by renal disease, with chronic kidney disease accounting for 843.6 million of them (Jager, et al., 2019). According to recent data, the global incidence and prevalence of chronic renal disease grew by 89 and $87 \%$ respectively, between 1990 and 2016. (Xie, et al., 2018).

According to the Centers for Disease Control and Prevention (CDC), chronic kidney disease affects 30 million people in the United States, or $15 \%$ of the adult population. End-stage renal disease (ESRD) has become more common in poor nations like Egypt. Diabetes mellitus and hypertension were thought to be the major causes of ESRD (Soliman, et al., 2012).
Chronic renal disease can be caused by a number of factors. Diabetes and/or hypertension, as well as old age, are the most common causes, but there are many others. These less prevalent causes of chronic kidney disease are normally diagnosed and treated at specialised renal clinics; nonetheless, non-renal physicians manage the majority of chronic kidney disease. (Rainey, 2018).

In the early stages of chronic kidney disease, there are usually no symptoms, but as the disease progresses, symptoms become more frequent and severe. As a result, it's most commonly detected through routine screening of persons who are at risk (such as those with diabetes or hypertension) or by coincidence when tests are needed for another reason. As a result, it is often identified by practice nurses in primary care (Rainey, 2018).

Renal replacement therapy, such as hemodialysis, continuous ambulatory peritoneal dialysis (CAPD), and kidney transplantation, is required for all patients with chronic kidney disease. (Rini, et al., 2021). To perform hemodialysis, however, the patient must have vascular access (Lok, et al., 2019).

The ideal hemodialysis vascular access is one that provides reliable, complication free access to deliver prescribed dialysis and that is also concurrently suitable for a given patient's needs (Huber, et al, 2020). 
Kidney disease is a major clinical and public health issue that affects people all over the world. Chronic kidney disease is associated with high health-care expenses, a poor quality of life, and catastrophic health consequences (including cardiovascular disease, kidney failure requiring kidney replacement therapy, infection, depression, and mortality) (Bello, et al., 2017).

Controlling risk factors and treating the disease to decrease its progression and lower the risk of complications can help to prevent chronic renal disease and its complications. To keep healthy kidneys, controlling those risk factors for chronic kidney disease that can be modified is critical for maintaining healthy kidneys (CDC, 2020).

Self-care is a coping approach for dealing with life's ups and downs that can help people gain independence and improve their quality of life (Banaye, et al., 2017). In chronic illness, self-care is a fundamental and integral part of treatment and patients who engage in self-care have significantly improved clinical outcomes, with better quality of life, fewer hospitalizations, and longer survival (Westland et al., 2016 \& Riegel et al., 2021).

Self-care encompasses a variety of behaviors, general and disease specific, in which persons suffering from a chronic illness engage to maintain their physical and emotional stability. These behaviors (e.g. assure sufficient sleep, take prescribed medications, manage stress, be physically active) are referred to as self-care maintenance (Barbaranelli et al., 2019).

Self-care interventions developed for specific patient groups are heterogeneous in the self-care behaviors targeted (e.g., diet, exercise, medication adherence), the mode of delivery, intervention intensity, and outcomes addressed (Jonkman et al., 2017).

Maintaining people's engagement in the care required by their health condition is crucial in any disease, especially a chronic one. Patients with chronic kidney disease who need hemodialysis therapy, which is usually done three times a week, are in this scenario. It necessitates a bevvy of other care considerations in order to maintain optimal compensation. These care actions involve changes in the eating habits, liquid intake, and adherence to HD, among others (Figueredo et al., 2014 \& Serra, et al., 2019). The preceding highlights the importance of teaching people self-care so that they accept full responsibility for their own health and participate actively in its upkeep. (Santana, et al., 2020)

Hemodialysis (HD) patients encounter a lot of difficulties with self-care. Patients must be able to control and manage their symptoms in order to improve self-care. Self-care behaviour can be enhanced with the help of education (Ramezani et al., 2019). Even though self-care for hemodialysis patients may require significant restrictions in lifestyle habits and behaviors, but participation in self-care behaviors may thus represent one way to mitigate adverse outcomes associated with CKD. (Wong, et al., 2018 \& Kim, et al., 2019).

Nurses can identify and manage risk factors to prevent or slow chronic kidney disease progression and they can provide patient-specific education to help patients take charge of their condition and promote well-being. Chronic kidney disease can result in several complications that will require treatment. In partnership with patients and physicians, nurses aid in the administration of treatment by assessing, planning, implementing, and evaluating care plans. (Chicca, 2020).

By identifying and controlling risk factors, nurses can help patients take control of their illness and promote well-being. They can also provide patient-specific education to assist patients take control of their health and improve their quality of life. Fluid overload, electrolyte imbalances, and anaemia are all complications of chronic renal disease that require treatment (Chicca, 2020).

\section{Significance of the study:}

Kidney disease has a significant impact on global health, as it is a leading cause of morbidity and mortality worldwide (Chronic Kidney Disease Collaboration, 2020). The total prevalence of dialysis patients in Egypt is 264 per million (ALmawsheki, et al, 2016). According to data and trends, the prevalence of end-stage renal illness is increasing over the world, particularly in developing nations, and in Egypt in particular. To promote patients' involvement and self-reliance in addressing their renal health problems and preventing complications, there is a need for sustained active patient education, support, and evaluation. So the researcher assessed the hemodialysis patients regarding their self care behavior.

Aim of study:

To assess self care behavior for hemodialysis patients.

Research Questions:

1. What is the level of knowledge of hemodialysis patients regarding self care behavior?

2. What is the level of practice of hemodialysis patients regarding self care behavior?

\section{Subject and Methods:}

Research design: A descriptive research design was adopted in this study.

Setting: The study was carried out in the dialysis unit of Beni-Suef University Hospital.

Sample: 55 patient who were attended the dialysis unit at Beni-Suef university hospital and meet the 
inclusion criteria were including in the study and they are agree to participate in the study.

Tools of the study:

The researcher created three tools to collect relevant data following reviewing of existing related literature in simple Arabic language, including the following:

Tool (I): A structured interviewing questionnaire form: It consisted of two parts:

First part: Assess the socio-demographic characteristics as (age, sex, marital status, income of family, residence and level of education, etc) (AbdEltwab scale, 2012).

Second part: it was included patient past history as hypertension, diabetes mellitus, chronic glomerulonephritis, etc.

Tool (2): knowledge assessment data sheet:

It was included (32)questions regarding patients' knowledge about kidney disease and dialysis it included(11)questions as (anatomy and physiology of kidney, definition, causes and sign and symptoms of kidney disease) , patients' knowledge about prescribed medication regimen and its complicationit included (4) questionsas (prescribed medication, importance of vitamin D and calcium and complication of prescribed medication), patients' knowledge about treatment routes of renal failure it included(6) questions as (ways of treatment, goal of dialysis, knowledge related to care of blood access site) and patients' knowledge about diet and exercises regimen related renal failure and dialysis it included(11)questions as (foods that contain low or rich potassium, importance of determining right amount of drinking water, weight measuring and doing exercises).

Scoring system for knowledge: The total knowledge score was 32 grads, with one grade for each correct answer and zero for each incorrect response. The scores of the items were added together for each area of knowledge, and the total was divided by the number of items, yielding a mean score for the part. These scores were transformed to a percent score as follows (Poor < 50percentage, Fair 50\% < 70\%, Good $>70 \%$ ). (Whdan, et al., 2019)

Tool (3): Hemodialysis patients' self-care measurement scale: This scale was created by (ShintaniKeiko, 2014) to assess the degree of universal selfcare and health-deviation requisites. The scale is divided into two parts: universal self-care and health-deviation self-care-behavior.

1.The universal self-care factor: consists of $\mathbf{5}$ factors and $\mathbf{3 5}$ requisites. These factors included patients' self-care about the dietary regulation: it was included (12) questions as (eat late at night, keep salt intake low, stop eating when you are approximately full, keep sugar intake low and always drink water after the bath), patients' self- care about stress prevention it included (9) questions as (avoid arguing and stressful confrontations, use methods to avoid getting angry and laugh away annoyances and insults), patients' self-care about food safety it included (5) questions as (check calories and sodium content when buying food, check the nutrition information panel when buying food, check the use-by date of food when shopping and check for lack of artificial ingredients in processed or kneaded foods), patients' self-care about exercise regulation it included (5) questions as (walk often, use a car to go short distances) and patients' selfcare about habit regulation it included (4) questions as (make time for prayer - religious activities, read books - watches movies, reduce or stop smoking, practice safe sex)

2.Health deviation self-care: consist of 3factors and 25requisites. These factors included shunt preservationfactor, therapeutic diet implementation factor, and theobservations of care instructions factor.

3.Patients' self-care about shunt preservation: it included ( 10)questions as (keep the shunt area clean, watching out for reddening pain or swellings, avoid carrying heavy weights with the arm used for blood shunting, avoid resting your head on the arm used for blood shunting), patients' self-care about therapeutic diet implementation it included (8) questions as ( keep to a physician's recommended water intake, keep salt intake to within the limits prescribed by the physician, keep calories to within the limits prescribed by the physician) and patients' self-care about the observations of care instructions it included (7) questions as (on specified days, measure the amount of urine produced, on specified days, preserve all urine produced during that day, avoid doing heavy labour with the arm used for blood shunting ).

Scoring system for Hemodialysis patients' selfcare measurement scale

Each item requisite was graded on a four-point scale, with 4 point being the very applicable, 3 point indicates somewhat applicable, 2 point denotes minimally applicable and 1 point $=$ not applicable at all.

The scores of the items were added up for each part, and the total was divided by the number of items, yielding a percent score. The level of universal selfcare of hemodialysis patients was deemed desirable if the scores were $50 \%$ or higher, while less than $50 \%$ was regarded undesirable. (Mahmoud et al., 2015)

Validity of tools: Following a reviewing of relevant literature, the study tools were created. Experts in family and community health nursing, as well as 
medical and surgical nursing, revised these tools for clarity, relevance, comprehensiveness, understanding, and applicability. The changes that were recommended were implemented.

Reliability: the researchers used reliability for testing the tool's internals by giving the identical tools to the same participants under similar conditions two times 15 days apart. Cronbach's alpha reliability was 0.848 for knowledge and 0.768 for the self-care measurement scale.

Methodology: Administrative phase:-

The head of the hemodialysis unit at Beni-Suef university hospital received official consent from the Dean of the Faculty of Nursing, Assiut University, to carry out the study, explaining the goal of the study and asking for permission to perform the study.

Pilot study: After following the developing of the study's required tool. A pilot study with $10 \%$ of patients was conducted to check that the generated tool was clear and applicable, as well as to estimate the time required to complete the questionnaire. Patients who took part in the pilot study were enrolled in the main study.

\section{Ethical consideration:}

The researcher followed all ethical issues in conducting the research. The research proposal was approved by the Ethical Committee of the Faculty of Nursing at Assiut University; there is no risk to study subjects during the application of the research; the study followed common ethical principles in clinical research; confidentiality and anonymity will be assured. Study subjects have the right to refuse to participate and/or withdraw from the study at any time for any reason; study subject privacy has been taken into account during data collection; and study subjects consent to engage in this study (oral agreement).

Field work:

An official letter approval was obtained from president of Beni-Suef University hospital to obtain necessary approval to conduct the study. This letter included a permission to collect the necessary data and explain the study's objective and scope. Each hemodialysis patients were informed about the purpose and nature of the study at hemodialysis unit. Data collection for this study took two months to complete from beginning of August 2019 till the September 2019 in dialysis unit at Beni-Suef university hospital, two days per week (Sunday and Wednesday), 3-4 patients per day. Every patient who agreed to participate in the study gave their oral agreement, and data confidentiality was ensured. After a thorough explanation, the researcher filled out the questionnaire for each patient, which took about 50-60 minutes.

\section{Statistical analysis:}

Data entry and data analysis were done using SPSS version 25 (Statistical Package for Social Science). Data were presented as number, percentage, mean, standard deviation. Chi-square test and Fisher Exact test were used to compare qualitative variables. Pvalue considered statistically significant when $\mathrm{P}<$ 0.05 . 


\section{Results:}

Table (1): Distribution of hemodialysis patients regarding to socio- demographic characteristics at Beni-Suef university hospital, 2018.

\begin{tabular}{|c|c|c|}
\hline Socio-characteristics & No(n=55) & $\%$ \\
\hline \multicolumn{3}{|l|}{ Age: (years) } \\
\hline$<20$ & 12 & 21.8 \\
\hline $20:<40$ & 20 & 36.4 \\
\hline $40:<60$ & 16 & 29.1 \\
\hline 60 and more & 7 & 12.7 \\
\hline \multicolumn{3}{|c|}{ Mean \pm SD38.61 \pm 6.32} \\
\hline \multicolumn{3}{|l|}{ Patients ' work } \\
\hline Governmental employee & 12 & 21.8 \\
\hline Free business & 17 & 30.9 \\
\hline Skilled worker & 8 & 14.5 \\
\hline Retired & 7 & 12.8 \\
\hline Not working / house wife & 11 & 20 \\
\hline \multicolumn{3}{|l|}{ Patients ' educational level } \\
\hline Illiterate & 19 & 34.6 \\
\hline Read \& write & 11 & 20 \\
\hline Primary & 7 & 12.7 \\
\hline Preparatory & 4 & 7.3 \\
\hline Secondary & 8 & 14.5 \\
\hline University & 6 & 10.9 \\
\hline \multicolumn{3}{|l|}{ Fathers' educational level } \\
\hline Illiterate & 26 & 47.3 \\
\hline Read \& write & 15 & 27.3 \\
\hline Primary & 2 & 3.6 \\
\hline Preparatory & 1 & 1.8 \\
\hline Secondary & 7 & 12.7 \\
\hline University & 4 & 7.3 \\
\hline \multicolumn{3}{|l|}{ Mothers' educational level } \\
\hline Illiterate & 21 & 38.2 \\
\hline Read \& Write & 10 & 18.2 \\
\hline Primary & 2 & 3.6 \\
\hline Preparatory & 3 & 5.5 \\
\hline Secondary & 10 & 18.2 \\
\hline University & 9 & 16.3 \\
\hline \multicolumn{3}{|l|}{ Fathers' work } \\
\hline Employee & 6 & 10.9 \\
\hline Free business & 30 & 54.5 \\
\hline Skilled worker & 14 & 25.6 \\
\hline Retired & 5 & 91 \\
\hline \multicolumn{3}{|l|}{ Mothers' work } \\
\hline Employee & 15 & 27.3 \\
\hline Housewife & 39 & 70.9 \\
\hline Retired & 1 & 1.8 \\
\hline \multicolumn{3}{|l|}{ Residence } \\
\hline Rural & 50 & 90.9 \\
\hline Urban & 5 & 9.1 \\
\hline \multicolumn{3}{|l|}{ Social status level } \\
\hline Low & 32 & 58.1 \\
\hline Moderate & 14 & 25.5 \\
\hline High & 9 & 16.4 \\
\hline
\end{tabular}


Table (2): Distribution of hemodialysis patients regarding to medical history at Beni-Suef university hospital, 2018.

\begin{tabular}{|c|c|c|}
\hline Medical history & No(55) & $\%$ \\
\hline \multicolumn{3}{|l|}{ 1.Presence of chronic diseases } \\
\hline Hypertension & 20 & 36.4 \\
\hline Diabetes mellitus & 13 & 23.6 \\
\hline Nephritis & 20 & 36.4 \\
\hline Others (e.g. SLE) & 2 & 3.6 \\
\hline \multicolumn{3}{|c|}{ 2.Taking medications for long periods as analgesics. } \\
\hline Yes & 35 & 63.6 \\
\hline No & 20 & 36.4 \\
\hline \multicolumn{3}{|c|}{ 3.Presence of chronic renal failure in your family members } \\
\hline Yes & 5 & 9.1 \\
\hline No & 50 & 90.9 \\
\hline \multicolumn{3}{|l|}{ 4.Smoking } \\
\hline Yes & 18 & 32.7 \\
\hline No & 37 & 67.3 \\
\hline \multicolumn{3}{|l|}{ 5.Time of chronic renal failure diagnosis } \\
\hline $1-3$ years & 20 & 36.4 \\
\hline 4-6 years & 20 & 36.4 \\
\hline $7-9$ years & 10 & 18.2 \\
\hline$>9$ years & 5 & 9.1 \\
\hline \multicolumn{3}{|l|}{ 6.Time of starting hemodialysis } \\
\hline $1-3$ years & 20 & $\mathbf{3 6 . 4}$ \\
\hline 4-6 years & 25 & 45.5 \\
\hline $7-9$ years & 10 & 18.2 \\
\hline \multicolumn{3}{|l|}{ 7.Number of hemodialysis sessions per week } \\
\hline Two & 0 & 0 \\
\hline Three & 55 & 100 \\
\hline \multicolumn{3}{|l|}{ 8.Number of hemodialysis hours per session } \\
\hline Three & 0 & 0 \\
\hline Four & 55 & $\mathbf{1 0 0}$ \\
\hline \multicolumn{3}{|l|}{ 9.Post hemodialysis session complains } \\
\hline Hypotension & 10 & 18.2 \\
\hline Restlessness & 15 & 27.3 \\
\hline Headache & 10 & 18.2 \\
\hline Muscle cramps & 15 & 27.3 \\
\hline Others (e.g.pain in the fistula) & 5 & 9.1 \\
\hline
\end{tabular}

Table (3): Distribution of hemodialysis patients regarding their knowledge about kidney and renal failure at Beni-Suef university hospital, 2018.

\begin{tabular}{|l|c|c|}
\hline \multicolumn{1}{|c|}{ Patients' knowledge } & N & \% \\
\hline $\begin{array}{l}\text { Anatomy of kidney: } \\
\text { Size of a normal human kidney }\end{array}$ & 10 & $\mathbf{1 8 . 2}$ \\
\hline $\begin{array}{l}\text { Physiology of kidney: } \\
\text { Functions of the kidneys }\end{array}$ & & \\
\hline $\begin{array}{l}\text { Definition of chronic renal failure: } \\
\text { A patient with chronic renal failure is one who has. }\end{array}$ & 6 & $\mathbf{1 0 . 9}$ \\
\hline Causes of chronic renal failure & 9 & $\mathbf{1 6 . 7}$ \\
\hline Signs and symptoms of renal failure & 3 & 5.4 \\
\hline Diagnosis of renal failure & 7 & 12.7 \\
\hline The necessary periodic medical analyzes for patients with renal failure & 2 & $\mathbf{3 . 6}$ \\
\hline Complications of renal failure & 5 & 9.1 \\
\hline Methods of reduction skin itching & 4 & 7.3 \\
\hline The types of blood connections & 12 & $\mathbf{2 1 . 8}$ \\
\hline
\end{tabular}




\begin{tabular}{|l|c|c|}
\hline \multicolumn{1}{|c|}{ Patients' knowledge } & N & \% \\
\hline Medications for renal failure & 12 & 21.8 \\
\hline The therapeutic medications were prescribed for hemdialysis patient & 20 & $\mathbf{3 6 . 4}$ \\
\hline Is it necessary to take vitamin D and calcium & 10 & 18.2 \\
\hline Importance of taking Vitamin "D" and calcium & 6 & 10.9 \\
\hline Complications /side effects of medications & 20 & $\mathbf{3 6 . 4}$ \\
\hline Methods of treatment renal failure & 11 & 20 \\
\hline Management renal failure & 8 & 14.6 \\
\hline Signs of inflammation of vascular access & 15 & $\mathbf{2 7 . 3}$ \\
\hline Methods of management of inflammation of vascular access & 10 & 18.2 \\
\hline Methods of Conservation of vascular access & 8 & 14.6 \\
\hline Coping with renal failure & 2 & $\mathbf{3 . 6}$ \\
\hline Nutrition and exercises & & 9 \\
\hline Foods low in potassium & 5 & 9 \\
\hline $\begin{array}{l}\text { Which of the following foods should be avoided because they contain a high } \\
\text { amount of potassium }\end{array}$ & 3 & 5.4 \\
\hline What should a patient with renal failure do if he feels low blood pressure & 15 & $\mathbf{2 7 . 3}$ \\
\hline What is the importance of exercise for hemodialysis patients? & 15 & 27.3 \\
\hline Your ideal weight is & \\
\hline What is the source of your information about renal Failure? &
\end{tabular}

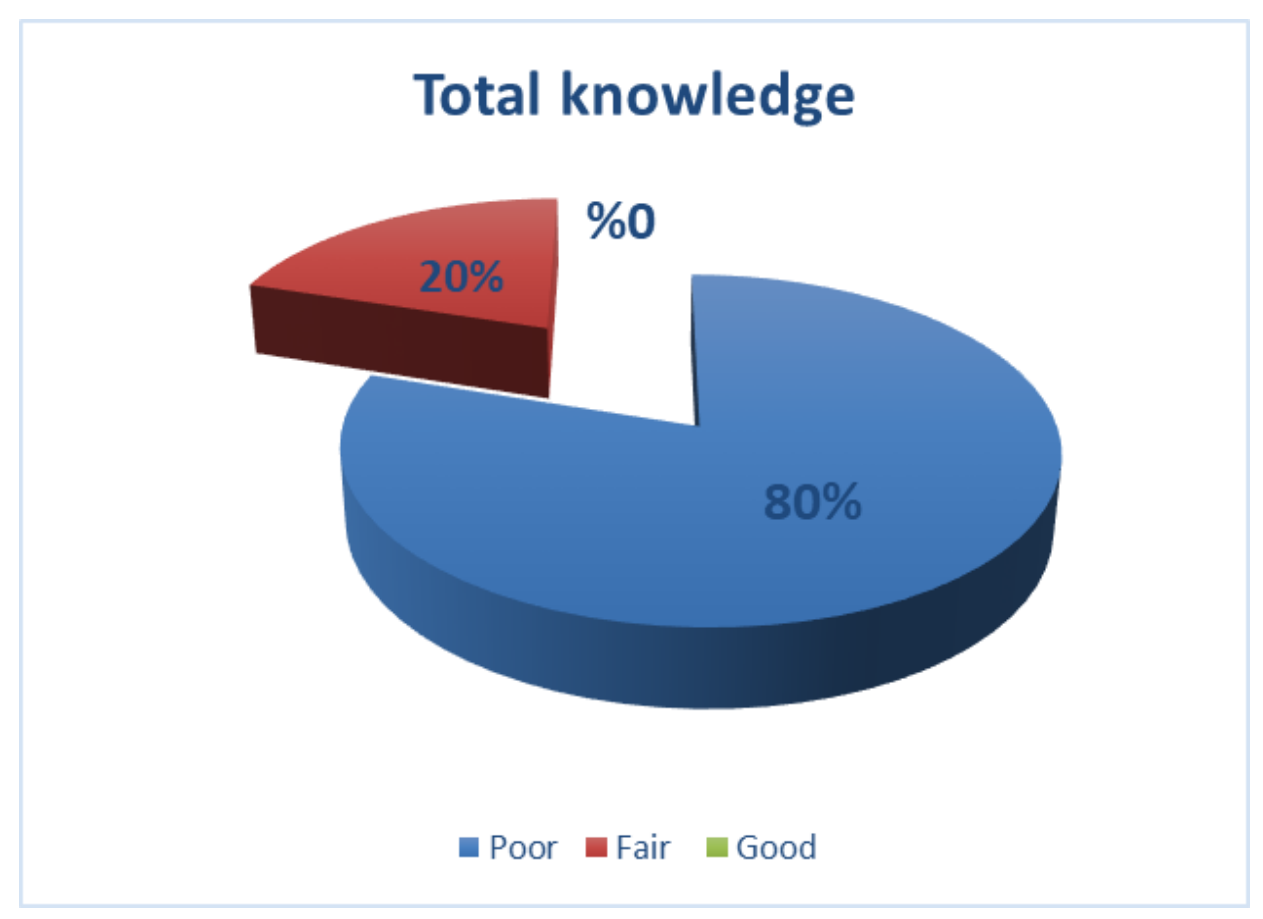

Figure (1): Distribution of hemodialysis patients according to their total level of knowledge at BeniSuef university hospital, 2018. 
Table (4): Distribution of hemodialysis patients regarding their practice level about universal selfcare for health behavior at Beni-Suef university hospital, 2018.

\begin{tabular}{|l|c|c|}
\hline \multicolumn{1}{|c|}{ Items } & N & \% \\
\hline Dietary regulation & 5 & 9.1 \\
\hline Eat late at night & 0 & $\mathbf{0 . 0}$ \\
\hline Keep salt intake low & 12 & 21.8 \\
\hline Stop eating when you are approximately full. & 10 & $\mathbf{1 8 . 2}$ \\
\hline Keep sugar intake low & 6 & 10.9 \\
\hline Always drink water after the bath & 6 & 10.9 \\
\hline Consume less-sweet foods & 0 & $\mathbf{0 . 0}$ \\
\hline Eat home-prepared meals & 6 & 10.9 \\
\hline Drink water daily & 8 & 14.6 \\
\hline Always drink water after exercise & 11 & $\mathbf{2 0}$ \\
\hline Keep fat intake low & 5 & 9.1 \\
\hline Eat snack between meals & 10 & $\mathbf{1 8 . 2}$ \\
\hline Avoid artificial ingredients(preservatives, chemical flavoring agents) & 4 & 7.3 \\
\hline Dietary food safety & 7 & 12.7 \\
\hline Check calories and sodium content when buying food & 5 & 9.1 \\
\hline Check the nutrition information panel when buying food & 9 & $\mathbf{1 6 . 7}$ \\
\hline Check the use-by date of food when shopping & 2 & $\mathbf{3 . 6}$ \\
\hline Check for lack of artificial ingredients in processed or kneaded foods & \multicolumn{2}{|c|}{} \\
\hline Take care to eat a balanced diet. & 3 & 5.4 \\
\hline Exercise regulation & 2 & $\mathbf{3 . 6}$ \\
\hline Walk often & 4 & 7.2 \\
\hline Use a car to go short distances & 12 & $\mathbf{2 1 . 8}$ \\
\hline Use stairs rather than the elevator / Use stairs to climb one or two floors & 5 & 9.1 \\
\hline Exercise daily (jogging, running, walking, radio calisthenics, or walking the dog) & \multicolumn{2}{|c|}{} \\
\hline Use the car, bus or train rather than walk, even for short distances & \multicolumn{2}{|c|}{} \\
\hline
\end{tabular}

Table (5): Distribution of hemodialysis patients regarding their practice level about universal selfcare for health behaviorat Beni-Suef university hospital, 2018.

\begin{tabular}{|l|c|c|}
\hline \multicolumn{1}{|c|}{ Items } & N & \multicolumn{2}{c|}{} \\
\hline Stress Regulation & 6 & 10.9 \\
\hline If made to feel unhappy, attempt to calm down as soon as possible & 5 & 9.1 \\
\hline Use methods to avoid getting angry & 0 & $\mathbf{0 . 0}$ \\
\hline Laugh away annoyances and insults & 2 & 3.6 \\
\hline Avoid arguing and stressful confrontations & 12 & 21.8 \\
\hline Remember that each person is individual, and do not get too angry & 7 & 12.7 \\
\hline If your health condition is pointed out by another person, take extra care & 12 & 21.8 \\
\hline Express your opinions without offending other people & 14 & $\mathbf{2 5 . 5}$ \\
\hline When feeling defeated, take a rest and try again & 10 & 18.2 \\
\hline Avoid lying and insulting people, to keep the mind & \multicolumn{2}{|c|}{} \\
\hline Habit regulation & 5 & 9.1 \\
\hline Make time for prayer - religious activities & 6 & 10.9 \\
\hline Read books / watches movies & 0 & $\mathbf{0 . 0}$ \\
\hline Reduce or stop smoking & 14 & $\mathbf{2 5 . 5}$ \\
\hline Practice safe sex & \multicolumn{2}{|c|}{} \\
\hline
\end{tabular}




\section{Total universal self care practice}

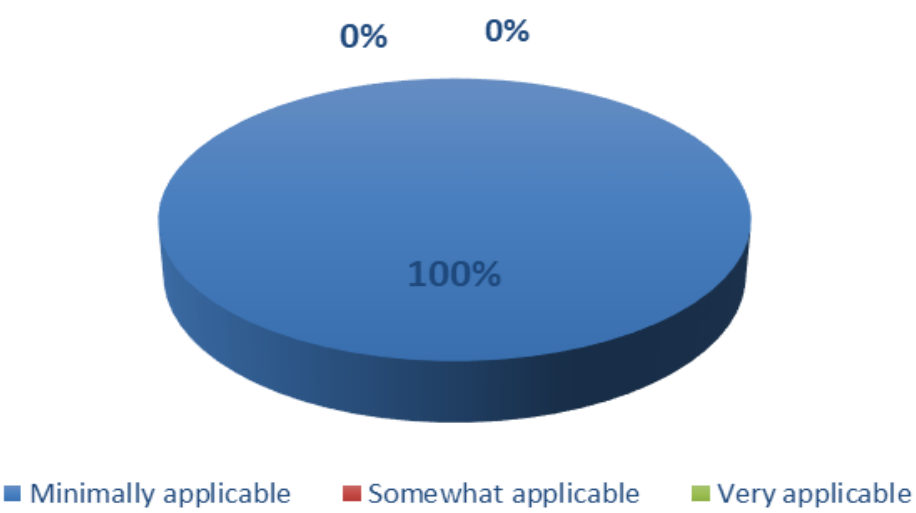

Figure (2): Distribution of the hemodialysis patients according to their total universal self-care practice at Beni-Suef university hospital, 2018.

Table (6): Distribution of hemodialysis patients regarding their practice level about health deviation self-care for health behavior at Beni-Suef university hospital, 2018.

\begin{tabular}{|l|c|c|}
\hline \multicolumn{2}{|c|}{ Items } & N \\
\hline Shunt preservation & 8 & 14.6 \\
\hline Keep the shunt area clean & 6 & 10.9 \\
\hline Watching out for reddening pain or swellings & 10 & $\mathbf{1 8 . 2}$ \\
\hline Avoid carrying heavy weights with the arm used for blood shunting & 12 & $\mathbf{2 1 . 8}$ \\
\hline Avoid resting your head on the arm used for blood shunting & 4 & 7.3 \\
\hline Avoid measuring blood pressure with the arm used for blood shunting & 5 & 9.1 \\
\hline Do not bear scratches on your skin & 0 & $\mathbf{0 . 0}$ \\
\hline Gargle every day in order to prevent colds & 14 & $\mathbf{2 5 . 5}$ \\
\hline Maintain distance when talking with people who have colds & 6 & 10.9 \\
\hline Wear a mask when colds are prevalent & 1 & 1.8 \\
\hline Avoid rubbing the area of the blood shunt or applying hot towels & & \\
\hline Therapeutic diet implementation & 15 & 27.3 \\
\hline Do you maintain the amount of water recommended by the doctor? & 14 & 25.5 \\
\hline Do you keep your salt intake within the limits recommended by your doctor? & 10 & 18.2 \\
\hline Do you maintain the food recommended by your doctor? & 12 & 21.8 \\
\hline Do you eat fruit according to your doctor's advice? & 11 & 20 \\
\hline Do you keep your calories within the limits recommended by your doctor? & 18 & $\mathbf{3 2 . 8}$ \\
\hline Do you keep the recommended diet for hemodialysis treatment? & 10 & 18.2 \\
\hline Do you keep your intake of sugar (sweet and rice) to a minimum? & 7 & 12.7 \\
\hline Do you eat a lot of foods that contain vitamins B and C? & 5 & $\mathbf{9 . 1}$ \\
\hline Do you avoid eating foods that you may be allergic to it? & & \\
\hline The observations of care instructions & 4 & 7.3 \\
\hline Measure the amount of urine produced & 2 & $\mathbf{3 . 6}$ \\
\hline Preserve all urine produced during that day & 10 & $\mathbf{1 8 . 2}$ \\
\hline Avoid doing heavy labor with the arm used for blood shunting & 5 & 9.1 \\
\hline Always undergo hemodialysis on the set days & 6 & 10.9 \\
\hline Consume medicine at times specified by the physician & 7 & 12.7 \\
\hline Avoid immersing the biopsy wound in a bath until it is healed & \\
\hline
\end{tabular}




\section{Total health deviation self -care pratice}

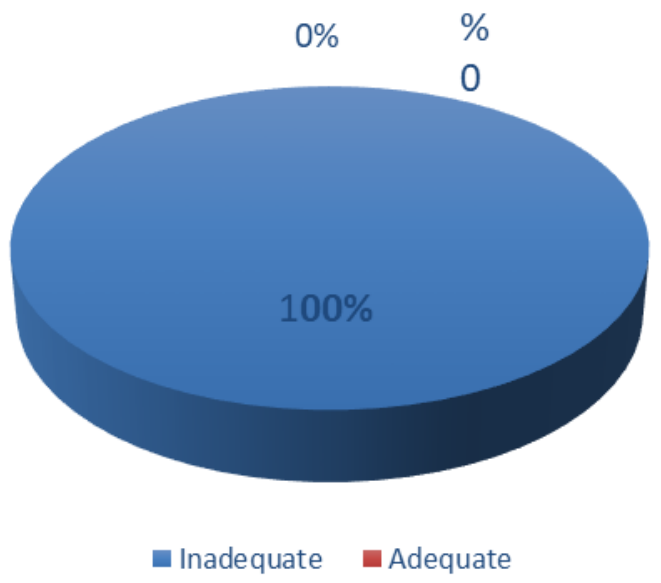

Figure (3): Distribution of the hemodialysis patients according to their total health deviation selfcare practice at Beni-Suef university hospital, 2018.

Table (7): Relation between socio- demographic characteristics of hemodialysis patients and their level of knowledge to kidney and renal failureat Beni-Suef university hospital, 2018.

\begin{tabular}{|c|c|c|c|c|c|c|c|c|c|}
\hline \multirow[t]{2}{*}{ Socio-characteristics } & \multirow[t]{2}{*}{$\mathbf{N}$} & \multicolumn{2}{|c|}{$\begin{array}{c}\text { Good } \\
\text { knowledge }\end{array}$} & \multicolumn{2}{|c|}{$\begin{array}{c}\text { Fair } \\
\text { knowledge }\end{array}$} & \multicolumn{2}{|c|}{$\begin{array}{c}\text { Poor } \\
\text { Knowledge }\end{array}$} & \multirow[t]{2}{*}{$\mathbf{X}^{2}$} & \multirow[t]{2}{*}{ p-Value } \\
\hline & & $\mathbf{N}$ & $\%$ & $\mathbf{N}$ & $\%$ & $\mathbf{N}$ & $\%$ & & \\
\hline \multicolumn{8}{|l|}{ Age: (years) } & \multirow{5}{*}{8.1} & \multirow{5}{*}{$.024 *$} \\
\hline$<20$ & 12 & 0 & 0.0 & 2 & 3.6 & 10 & 18.2 & & \\
\hline $20:<40$ & 20 & 0 & 0.0 & 6 & 10.9 & 14 & 25.5 & & \\
\hline $40:<60$ & 16 & 0 & 0.0 & 2 & 3.6 & 14 & 25.5 & & \\
\hline 60 and more & 7 & 0 & 0.0 & 1 & 1.8 & 6 & 10.9 & & \\
\hline \multicolumn{8}{|l|}{ Patients ' work } & \multirow{6}{*}{11.6} & \multirow{6}{*}{$.006^{*}$} \\
\hline Governmental employee & 12 & 0 & 0.0 & 3 & 5.5 & 9 & 16.4 & & \\
\hline Free business & 17 & 0 & 0.0 & 5 & 9.1 & 12 & 21.8 & & \\
\hline Skilled worker & 8 & 0 & 0.0 & 1 & 1.8 & 7 & 12.7 & & \\
\hline Retired & 7 & 0 & 0.0 & 0 & 0 & 7 & 12.7 & & \\
\hline Not working / house wife & 11 & 0 & 0.0 & 2 & 3.6 & 9 & 16.4 & & \\
\hline \multicolumn{8}{|l|}{ Patients ' educational level } & \multirow{7}{*}{42.7} & \multirow{7}{*}{$.000 * *$} \\
\hline Illiterate & 19 & 0 & 0.0 & 4 & 7.3 & 15 & 27.3 & & \\
\hline Read \& write & 11 & 0 & 0.0 & 1 & 1.8 & 10 & 18.2 & & \\
\hline Primary & 7 & 0 & 0.0 & 3 & 5.5 & 4 & 7.3 & & \\
\hline Preparatory & 4 & 0 & 0.0 & 2 & 3.6 & 2 & 3.6 & & \\
\hline Secondary & 8 & 0 & 0.0 & 0.0 & 0.0 & 8 & 14.5 & & \\
\hline University & 6 & 0 & 0.0 & 1 & 1.8 & 5 & 9.1 & & \\
\hline \multicolumn{8}{|l|}{ Residence } & \multirow{3}{*}{39.4} & \multirow{3}{*}{$.000 * *$} \\
\hline Rural & 50 & 0 & 0.0 & 9 & 16.4 & 41 & 74.6 & & \\
\hline Urban & 5 & 0 & 0.0 & 2 & 3.6 & 3 & 5.5 & & \\
\hline \multicolumn{8}{|l|}{ Social status level } & \multirow{4}{*}{16.8} & \multirow{4}{*}{$.000 * *$} \\
\hline Low & 32 & 0 & 0.0 & 7 & 12.6 & 25 & 45 & & \\
\hline Moderate & 14 & 0 & 0.0 & 3 & 5.5 & 11 & 20 & & \\
\hline High & 9 & 0 & 0.0 & 1 & 1.8 & 8 & 14.6 & & \\
\hline
\end{tabular}

(*) Statistically significant correlation at P-value $<0.05$ 
Table (8): The relation between socio-demographic characteristics of hemodialysis patients and their level of practice regarding self-care for health behavior at Beni-Suef university hospital, 2018.

\begin{tabular}{|c|c|c|c|c|c|c|c|c|c|}
\hline \multirow[t]{2}{*}{ Socio-characteristics } & \multirow[t]{2}{*}{$\mathbf{N}$} & \multicolumn{2}{|c|}{$\begin{array}{c}\text { Very } \\
\text { applicable }\end{array}$} & \multicolumn{2}{|c|}{$\begin{array}{l}\text { Somewhat } \\
\text { applicable }\end{array}$} & \multicolumn{2}{|c|}{$\begin{array}{l}\text { Minimally } \\
\text { applicable }\end{array}$} & \multirow[t]{2}{*}{$\mathbf{X}^{2}$} & \multirow[t]{2}{*}{ p-Value } \\
\hline & & $\mathbf{N}$ & $\%$ & $\mathbf{N}$ & $\%$ & $\mathbf{N}$ & $\%$ & & \\
\hline \multicolumn{8}{|l|}{ Age: (years) } & \multirow{5}{*}{14.2} & \multirow{5}{*}{$.038 *$} \\
\hline$<20$ & 12 & 0 & 0.0 & 1 & 1.8 & 4 & 7.3 & & \\
\hline $20:<40$ & 20 & 0 & 0.0 & 6 & 10.9 & 29 & 52.7 & & \\
\hline $40:<60$ & 16 & 0 & 0.0 & 1 & 1.8 & 9 & 16.4 & & \\
\hline 60 and more & 7 & 0 & 0.0 & 1 & 1.8 & 4 & 7.3 & & \\
\hline \multicolumn{8}{|l|}{ Patients ' work } & \multirow{6}{*}{12.6} & \multirow{6}{*}{$.011^{*}$} \\
\hline Governmental employee & 12 & 0 & 0.0 & 6 & 10.9 & 29 & 52.7 & & \\
\hline Free business & 17 & 0 & 0.0 & 4 & 7.3 & 11 & 20 & & \\
\hline Skilled worker & 8 & 0 & 0.0 & 2 & 3.6 & 2 & 3.6 & & \\
\hline Retired & 7 & 0 & 0.0 & 0 & 0 & 8 & 14.5 & & \\
\hline Not working / house wife & 11 & 0 & 0.0 & 1 & 1.8 & 5 & 9.1 & & \\
\hline \multicolumn{8}{|l|}{ Patients ' educational level } & \multirow{7}{*}{26.9} & \multirow{7}{*}{$.005^{* *}$} \\
\hline Illiterate & 19 & 0 & 0.0 & 4 & 7.3 & 15 & 27.3 & & \\
\hline Read \& write & 11 & 0 & 0.0 & 1 & 1.8 & 10 & 18.2 & & \\
\hline Primary & 7 & 0 & 0.0 & 1 & 1.8 & 4 & 7.3 & & \\
\hline Preparatory & 4 & 0 & 0.0 & 6 & 10.9 & 29 & 52.7 & & \\
\hline Secondary & 8 & 0 & 0.0 & 4 & 7.3 & 11 & 20 & & \\
\hline University & 6 & 0 & 0.0 & 1 & 1.8 & 5 & 9.1 & & \\
\hline \multicolumn{8}{|l|}{ Residence } & \multirow{3}{*}{28.4} & \multirow{3}{*}{$.000 * *$} \\
\hline Rural & 50 & 0 & 0.0 & 9 & 16.4 & 41 & 74.6 & & \\
\hline Urban & 5 & 0 & 0.0 & 2 & 3.6 & 3 & 5.5 & & \\
\hline \multicolumn{8}{|l|}{ Social status level } & \multirow{4}{*}{18.3} & \multirow{4}{*}{$.027 * *$} \\
\hline Low & 32 & 0 & 0.0 & 3 & 5.5 & 2 & 3.6 & & \\
\hline Moderate & 14 & 0 & 0.0 & 9 & 16.4 & 41 & 74.6 & & \\
\hline High & 9 & 0 & 0.0 & 2 & 3.6 & 8 & 14.6 & & \\
\hline
\end{tabular}

(*) Statistically significant correlation at $P$-value $<0.05$

Table (9): Correlation between total knowledge of hemodialysis patients with their universal selfcare and their health deviation self-care for health behaviorat Beni-Suef university hospital, 2018.

\begin{tabular}{|l|c|c|c|c|}
\hline \multicolumn{2}{|c|}{ Item } & $\begin{array}{c}\text { Total } \\
\text { knowledge }\end{array}$ & $\begin{array}{c}\text { Universal self- } \\
\text { care behavior }\end{array}$ & $\begin{array}{c}\text { Health deviation } \\
\text { self-care behavior }\end{array}$ \\
\hline \multirow{2}{*}{ Total knowledge } & $\mathrm{r}$ & 1 & .704 & .764 \\
\cline { 2 - 5 } & $\mathrm{p}$ & - & $.000^{* *}$ & $.000^{* *}$ \\
\hline \multirow{2}{*}{ Universal self-care for health behavior. } & $\mathrm{r}$ & .704 & 1 & .836 \\
\cline { 2 - 5 } & $\mathrm{p}$ & $.000^{* *}$ & - & $.000^{* *}$ \\
\hline $\begin{array}{l}\text { Health deviation self-care for health } \\
\text { behavior. }\end{array}$ & $\mathrm{r}$ & .764 & .836 & 1 \\
\cline { 2 - 5 }
\end{tabular}

(*) Statistically significant correlation at $P$-value $<0.05$

Table (1): Shows that the age group $20:<40$ years represents $36.4 \%$ with mean age \pm SD $38.61 \pm 6.32$. It was observed also, 30.9\%, 34.6\%, 90.9\%,58.1\% and $16.4 \%$ respectively of them were working free business, were illiterate, were from rural area and had a low economic standard.

Table (2): Illustrates that $36.4 \%$ of the hemodialysis (HD) patients had hypertension and nephritis respectively that may causes chronic renal failure. it was observed also that $90.9 \%$ of them hadn't family history. $67.3 \%$ of them were non smoker. According totime of chronic renal failure diagnosis, it was observed that $36.4 \%$ of them had diagnosed from 1-3 years and 4-6 years respectively.It was also revealed that $36.4 \%$ had diagnosed from 1-3 years and $36.4 \%$ of HD patients were starting hemodialysis from one year to 3 years and $100 \%$ of HD patients mentioned that they were scheduled for three times a week and four hours per session.

Table (3): Clears HD patients' knowledge about kidney and renal failure. It was observed that $18.2 \%$ of HD patients known size of a normal humankidney, while $10.9 \%$ of them known functions of the kidneys. Also this table shows that $16.7 \%$ of HD 
patients known definition of chronic renal failure. Regarding to diagnostic methods of renal failure, it was noticed that $3.6 \%, 21.8 \%$ and $27.3 \%$ respectively of them known diagnostic methods of renal failure,methods of reduction skin itching and known ideal weight.

Figure (1): It was found that $80 \%$ of HD patients had poor knowledge score.

Table (4): Indicates that $18.2 \%$ of them avoid artificial ingredients (preservatives, chemical flavoring agents). No one of them kept salt intake low or eat home-prepared meals, while $20 \%$ of them keep fat intake low. Also shows that, $16.7 \%$ of HD patients check for lack of artificial ingredients in processed or kneaded foods and $3.6 \%$ of them take care to eat a balanced diet and do not use a car to go short distances.

Table (5): Shows that no one of the HD patients laugh away annoyances and insults and $25.5 \%$ of them when feeling defeated, take a rest and try again.it was noticed that no one of the HD patients reduce or stop smoking and $25.5 \%$ of them practice safe sex.

Figure (2): It was found that $100 \%$ of HD patients had minimal applicable practice of universal selfcare for health behavior.

Table (6): It was found that $21.8 \%$ of HD patients avoid resting your head on the arm used for blood shunting and $18.2 \%$ of them avoid carrying heavy weights with the arm used for blood shunting. Also, $25.5 \%$ of themmaintain distance when talking with people who have colds. As regard to therapeutic diet implementation, $32.8 \%$ of them keep the recommended diet for hemodialysis treatment and 9.1 $\%$ of them avoid eating foods that you may be allergic. According to the observations of care instructions, also $3.6 \%$ of them preserve all urine produced during that day and $18.2 \%$ of them avoid doing heavy labor with the arm used for blood shunting.

Figure (3): It was found that $100 \%$ of HD patients had inadequatehealth-deviation practice regarding health behavior.

Table (7): Revealed that there was a significant statistical difference between knowledge of HD patients regarding kidney and renal failure, HD patients' age at $\mathrm{P}=.024$ and work at $\mathrm{P}=.006$, education level, their residence and social status standard at $\mathrm{P}=0.000$.

Table (8): Revealed that there was a significant statistical difference between of practice regarding self-care for health behavior of HD patients, HD patients' age and work, education level, their residence and social status standard at $\mathrm{P}=0.038,0.011,0.05,0.000$ and 0.027 respectively.
Table (9): Revealed that there was a significant statistical difference between HD patients'totalknowledgeand their universal self-care and health deviation self-care for health behavior at $\mathrm{P}=0.000$.

\section{Discussion:}

Chronic kidney disease is regarded as a public health issue worldwide, with an estimated 50 million individuals suffering from the disease. (Bilal et al, 2020). Despite developments in renal transplant and peritoneal dialysis modalities, hemodialysis (HD) remains the most extensively used renal replacement therapy. (Islam, et al., 2017).

The present study aimed to assess self-care behavior for hemodialysis patients.

The present study shows that the age range varied from less than 20yrs to more than $60 \mathrm{yrs}$ and it is observed that chronic kidney disease affects not only the elderly, who were considered as risk group, but also young and middle-aged people, while more than of one third of HD were age $20:<40$. These results agree with Clementino, et al.,(2018) who conducted a study about hemodialysis patients: the importance of self-care with the arteriovenous fistula, Brazil and they reported that the age of HD patients ranged from 19 : more than 60 years and one quarter of HD were age 19 : 40. Anjos et al., 2013, \& Pereira, et al., 2015 mentioned that the structure and function of the kidneys deteriorate with age, and the glomerular filtration rate (GFR) slowly decreases with age, declining by $25 \%$ by the age of 40 . Furthermore, there is a decline in kidney weight and volume, which is related with the usual comorbidities of the third age, which causes an increase in the vulnerability of the renal system, which results in a loss of the kidneys' internal equilibrium, putting these people at a higher risk of renal impairment.

The present study revealed that approximately one third of HD patients were working free business. This finding agree with Ramezani et al., (2019) who conducted study about effect of educational intervention on promoting self-care in hemodialysis patients, Iran and reported that less than one quarter of intervention group of hemodialysis patients were working free job. In contrast with the present study, another study conducted by Clementino et al., (2018) and they reported that vast majority of patients were unemployed. Constraints develop as a result of changes in physical capacity; HD treatments, which occur three times per week and last four hours (excluding travel time to and from the dialysis centre); physical disorders, and the need for self-care. All this will be obstacles to routine work. 
According to patients ' educational level, the present study showed that less than one quarter were illiterate while more than one third were had higher education. These results disagree with Mosavi et al., (2020) who study effect of education based on "PRECEDE" model on self-care behavior in hemodialysis patients, Lordegan Hospital, Iran and reported that more than three quarters of intervention group of hemodailysis patients are illiterate and no one have higher education. The level of education is a critical aspect, as higher education provides greater access to knowledge and improves economic conditions. On the other hand, illiteracy or even a lack of education might obstruct the learning of self-care and adherence to healthy lifestyle practices.

Regarding residence, the current study results show that vast majority of HD patients were living in rural area. The result findings were in contrast with Mahjubian et al., (2018) they conducted study about effects of group discussion based education on the promotion of self-management behaviors in hemodialysis patients, Iran and reported that that vast majority of HD patients were living at urban area.

Concerning the economic situation, only more than one tenth had a high economic standard and themajority of the HD patients is in poor to moderate standard. The study findings stand in the line with Gerogianni et al., (2016)they conducted study about social life of patients undergoing haemodialysis in four hospitals in Athens and reported that only around one tenth have a very good and excellent economic standard respectively and the economic status of more than half of the of the HD patients is in poor to moderate status. It can be paired with the fact that an increase in medical-hospital expenditures, age, and associated health problems (heart, hypertension, diabetes mellitus, anaemia) all have a detrimental impact on economic standards and produce financial challenges for the majority of HD patients.

According to the presence of chronic diseases that may cause of chronic renal failure in hemodialysis patients, the current study indicated that the most common chronic diseases of hemodialysis patients were glomerulonephritis and hypertension followed by diabetes, respectively that can causes of chronic renal failure. This finding in accordance with Yang, et al, (2019) they conducted study about self-care behavior of hemodialysis patients with arteriovenous fistula in china: a multicenter, cross-sectional study and reported that chronic glomerulonephritis was the most common form of primary cause of end-stage renal disease, followed by hypertensive nephropathy and diabetic nephropathy. Ministry of Health Democratic Republic of the Congo, (2014) \& Guimarães et al., (2016) mentioned that Both hypertension and diabetes promote a slow and progressive deterioration of target organs and tissues, including the kidneys, in distinct ways.

The current study showed that less than two thirds of HD patients were taking regular medications and vast majority hadn't family history of chronic renal failure. The current study result was consistence with ZainELdin et al., (2018) they study effect of selfcare model intervention on quality of life of children undergoing hemodialysis, at Menoufia University Hospital, Shebin El-kom city and found that the highest percentages of studied patients hadn't family history of kidney problems and also, it was found that the majority of HD patients get medication for long period.

The present study indicated that majority of $\mathrm{HD}$ patients were non smoker. This result in same line with Ramezani et al., (2019) reported that two thirds of intervention group of hemodialysis patients are non smoker and just more than one tenth are smoker.

In addition, the present results show that the dialysis duration of less than one fifth of HD patients were from one year to 3 years. This result was consistent with Ikiz et al.,(2020) who conducted a study about validation of the scale of assessment of self-care behaviours for arteriovenous fistula in patients ongoing haemodialysis in Turkey and they reported that the dialysis duration of around one fifth of HD patients were from one year to 3 years.

According to number of number of hemodialysis sessions per week, the current study demonstrated that all HD patients were scheduled for three times a week. The resultfindings was in same line with Mahmoud et al., (2014) they conducted a study about assessment of self-care practice of patients on maintenance hemodialysis at Cairo University hospitals and reported that vast majority of HD patients were scheduled for three times a week.

Also, the current study indicated that the most common symptoms that HD patients were complaining restlessness and muscle cramps. The current study result was consistence with Pretto et al., (2020) they conducted study about quality of life of chronic kidney patients on hemodialysis and related factors in Rio Grande do Sul, Brazil and they found that around one half of the patients reported restlessness after the end of the session.

Considering HD patients' knowledge regarding renal failure revealed that, the majority of them had poor knowledge related to kidney anatomy and physiology and diagnosis of renal failure. The study results were consistence with Whdan et al., (2019) who study home self-care for client with hemodialysis at Ashmon district Hospital, Menofia governorate and found that no one had good knowledge about kidney anatomy and physiology and diagnosis of renal failure. The poor level of client's knowledge may be 
due to the health care personnel didn't give the necessary and required knowledge to these clients.

As regarding methods of conservation of vascular access, less than one third of HD patients knew methods of conservation of vascular access. The current study result wasn't consistence with Ozen et al., (2017) who study investigation of the knowledge and attitudes of patients who are undergoing hemodialysis treatment regarding their arteriovenous fistula (AVF) and reported that majority of patients showed having information on AVF care. It is critical to provide structured and systematic information on AVF management to the patient Also, it is also vital to recognise the need to understand the importance of maintaining proper self-care behaviour. In order to verify, maintain and preserve vascular access functionality.

In addition, the current study demonstrated that less than one tenth of HD patients knew foods should be avoided because they contain a high amount of potassium. The present study results concur with Hafezieh, et al, (2020) they conducted study about self-management, self-efficacy and knowledge among patients under haemodialysis: a case in Iran and reported that less than one tenth of patients had good knowledge of the phosphate, potassium, and sodium content of various foods, despite the fact that too much of each of these minerals can have negative consequences. This indicates little insight into the motivation for the restrictions.

The current study indicated that majority of HD had poor total knowledge. The results of present study was consistence with Whdanet al., (2019) found that more than half of patients had poor knowledge.

According to dietary regulation, the current study results show that less than one quarter of HD patients keep fat intake low and less than two tenth of HD patients reported keep sugar intake low. The result findings were in agreement with (Wong et al., 2018) they conducted study about association between health literacy and self-care behaviors among patients with chronic kidney disease, San Francisco, USA and found that less than two tenth of patients reported frequent fast food consumption that contain high fat and salt, while more than half of the study participants said they consume sugary beverages on a daily or weekly basis. It could be attributable to an increase in the number of illiterate patients and patients from rural areas, who have less knowledge and adhere to a proper diet.

Regarding to exercise regulation, it is surprising that the results explain that highest percentage of patients were active, around one quarter of the HD patients practice physical exercise than using motor vehicle (bus, care, etc) even for short distance. It is expected that they were inactive related to complication hemodialysis and spending more time lying down or patients are afraid from practice exercise. It cannot be denied that on dialysis days, sedentary behaviour was more pronounced, but vast majority of HD patients were living in rural areas that is characterized by active lifestyle from going to the field and animals and birds breeding, etc. while urban is characterized by sedentary lifestyle and may HD patients were aware general benefits of exercises. This result is similar to Dungey et al., (2017) who conduct study about exercise during haemodialysis promotes an anti-inflammatory leucocyte profile and reported that in comparison to healthy counterparts in the general population, patients were less physically active. On the contrary by El Sayed, (2018) who study Assessment of self-care behaviors, self-efficacy and level of physical activity of patients undergoing hemodialysis at hemodialysis center at hospital affiliated to Ministry of Health (Port Said General Hospital) that sited in Port Said city and reported that more than half of patients had inadequate physical level.

Concerning to stress prevention and habit regulation the result explained that no one use a humor (laugh away in stressful situations or reduce or stop smoking. This result is similar to Parvanet al. (2015) they evaluate the coping strategies to stress among patients undergoing hemodialysis (HD) and peritoneal dialysis (PD) at the Imam Reza Educational-Medical Hospital they found among the ten most frequently utilized components, the greatest coping approach in both groups was 'is trying to preserve a sense of humour'. Also, The result findings were in agreement with (Wong, et al., 2018) found that nearly one-third of patients reported current tobacco use. This reflect that patients don't have the capacity to absorb the bad feelings and coping with stressful situations, however laughter and humour are one sort of coping with stress that reduces the harshness of stressful events, provides a sense of strength, fairness, and rest, and encourages people to keep going in life.

According to shunt preservation, the current study revealed that around two tenth of HD patients avoid resting head on the blood shunting arm, and just two tenths of them and less than two tenth of them avoid carrying heavy weights with the arm that is used for blood shunting. These results were disagreement with Rashid et al., (2018) who conducted study about Arteriovenous fistula (AVF) self-care: A study at a Tertiary Care Hospital in Lahore, Pakistan and they found around vast majority the patients were always avoiding sleep over AVF access site and weight lifting avoidance from AVF access site.

Concerning to therapeutic diet implementation, the result reflect that around one third of HD patients 
keep the recommended diet for hemodialysis treatment. These results were agreement with Santana et al., (2020) who conducted study about Self-care in individuals with chronic kidney disease on hemodialysis, Chile and reported that the majority of patients reported that they could eat whatever they wanted but with certain restrictions. This could be because they are aware of the risks of non-adherence, such as uremia, muscle pains, poor wound healing, poorly managed blood pressure, and extreme thirst.

According to the observations of care instructions, the current study revealed that less than one tenth of HD patients preserve all urine produced during day.These results were agreement with Rachmadi et al., (2020) who conducted study about relationship between self-care for fluid limitation and interdialytic weight gain among patients with hemodialysis at ratuzalecha hospital, Martapura and reported that most of the patients did not calculate the fluid intake and urine output a day. keeping adequate fluid intake in a day is important to avoid complications such as shortness breath, hypertension, and pulmonary edema. Fluid restriction is necessary to provide comfort to patients before and after hemodialysis; nevertheless, low desire to diet and fluid restriction may be a barrier to self-care management among hemodialysis patients. The present study revealed that all of HD patients had poor and inadequate universal and health deviation self-care practice for health behavior. These results was consistence with Rahimi et al., (2017) they conducted study about the effect of self-care on patients undergoing hemodialysis in the Sanandaj hospitals affiliated to Kurdistan University of Medical Sciences and found around less than two thirds of patients in the intervention group before the intervention were independent and practice of most patients were insufficient. Hemodialysis patients face a variety of issues and dramatic lifestyle changes as a result of their numerous and sophisticated pharmacological regimens, which may have an impact on their social and psychological functioning. Furthermore, treatment that does not include the patient's participation and some self-care tasks is ineffective in reaching the intended effects.

The present study cleared that there was statistical significant difference between HD patients' age and work, education level and their level knowledge to kidney and renal failure. This result was in contrast with Whdan et al., (2019) who showed that no significant correlation was observed between age and occupation with patient's knowledge about renal failure . On the other hand, significant correlation was detected between education levels and knowledge about renal failure, knowledge related to diet regimen, knowledge related to diet regimen and fluid and vascular access and medication for client HD.
Concerning the relation between socio demographic characteristics and universal self-care behavior study findings indicated that; there were highly significant differences between universal self-care behaviors of studied patients and their age, work, and educational level this result agreed with Wang,et al., (2019) who conducted study about patient assessment of chronic kidney disease self-care using the chronic kidney disease scale in Taiwan and reported that self-care behavior was correlated with age, sex, and education level.

Current results suggested that there is a close association between self-care behavior and disease knowledge in patients with chronic kidney disease. Also, cleared that there was statistical significant difference between HD patient's level knowledge and their health deviation self-care for health behavior. The current finding was in agreement with Tsai et al., (2021) they conducted study about the interaction between self-care behavior and disease knowledge on the decline in renal function in chronic kidney disease, Taiwan and found that self-care behavior was positively and significantly correlated with disease knowledge and showed that high disease knowledge scores were significantly associated with high selfcare behavior scores. Poor disease knowledge contributes to inadequate self-care behavior, and both of them are barriers to efficient chronic kidney disease care. Given the silent nature of chronic kidney disease, it's critical to grasp the fundamentals of kidney function, disease progression symptoms, and disease status. Due to a lack of disease information, joint decision-making for the therapeutic plan may be challenging. Understanding the determinants of disease knowledge and self-care behaviour is therefore critical for chronic renal disease patients.

\section{Conclusion:}

The current study concluded that the majority of hemodialysis patients had poor knowledge level and all patients had poor practice regarding self care behavior.

\section{Recommendation:}

The study advised that, based on the findings:

1- Establishing an educational program for hemodialysis patients regarding self-care behavior. Programs should be continuous and repeated, and refresher courses for assure they have adequate knowledge level and satisfactory level of practiceofself care behavior should be every definite period.

2- Design instruction model for nurses to follow up periodically with hemodialysis patients about practicing self-care behavior.

3- The care provider should reward committed hemodialysis patients to encourage others. 
4- Set up a counseling room in the HD units provided with needed resources to enhance selfcare practice for $\mathrm{HD}$ patients with appropriate referral system

5- Reapplication of the study on a large sample from different hospitals as well as from different geographical area in Egypt to evaluate the effect of hemodialysis on self-care behavior.

6- A simple manual of guidelines of care for patients undergoing hemodialysis should be available in all units to be provided to newly admitted patients.

7- Develop and coordinate multidisciplinary team approach in the hemodialysis unit that include the primary nurses, renal physicians, social workers, dietitians, psychotherapists and physiotherapists to assist patients in maintaining near normal life style at the highest possible level.

\section{References}

- AbdEltwab. (2012): Ocioeconomic scale, Faculty of Education. Assiut University.

- Barbaranelli C, Riegel B, Carlson B, Sethares KA, Daus M, Moser DK, Miller J, Osokpo OH, Lee S, Brown S, \&Vellone E (2019): Psychometric Testing of the Revised Self-Care of Heart Failure Index. J Cardiovasc Nurs. 2019; 34(2):183-192.

- Bilal A, Zaman F, Abrar S, Rahim S, Hussain M, \& Shah S (2020): Effect of age and gender on acute copplications of hemodialysis in patients with chronic kidney disease secondary to diabetes mellitus. Pakistan Armed Forces Medical Journal.2020; 70(3):781-786.

- Centers for Disease Control and Prevention (2020): Chronic Kidney Disease Initiative : Prevention \& Risk Management, 2020. Available at https://www.cdc.gov/kidneydisease/preventionrisk.html.

- Chicca J (2020): Adults with chronic kidney disease: Overview and nursing care goals. American Nurse Journal , 2020; 15 (3)16-23.

- Chronic Kidney Disease Collaboration (GBD) (2020): Global, regional, and national burden of chronic kidney disease, 1990-2017: a systematic analysis for the Global Burden of Disease Study 2017. Lancet. 2020; 395(10225):709-733.

- Clementino D, Souza A, Barros D, Carvalho D, Santos C, \& Fraga S (2018). Hemodialysis patients: the importance of self-Care with the arteriovenous fistula.. J Nurs UFPE .2018; 12(7):1841-52.

- Costa M, Sampaio J, Teixeira O, Pinheiro M, Leite E, \& Pereira A (2015): Renal disease: social, clinical and therapeutic profile of the elderly attended in a nephrology service. Espaç. saúde (Online). 2015 Apr/June;16(2):77-85
- Crews D, Bello A, \& Saadi G (2018): Burden, access, and disparities in kidney disease. Nephron. 2018; 2:1-8

- Dungey M, Young H, Churchward D, Burton J, Smith A, \& Bishop N (2017): Regular exercise during haemodialysis promotes an antiinflammatory leucocyte profile. Clin Kidney J. 2017;10 (6):813-821.

- El Sayed S (2018): Assessment of Self-Care Behaviors, Self-Efficacy and Level of Physical Activity of Patients Undergoing Hemodialysis at hemodialysis center. Port Said Scientific Journal of Nursing (PSJN) .2018; 1(5) 73-94

- Gerogianni S, Babatsikou F, Gerogianni G , Koutis C, Panagiotou M, \& Psimenou E (2016): Social Life of Patients Undergoing Haemodialysis.. International Journal of Caring Sciences. 2016; 9. 122-134.

- Guimarães G, Goveia V, Mendonza I, Corrêa A, Matos S, \& Guimarães J (2016): Profile of the patient usinge a central venous catheter during hemodialysis. Rev enferm UFPE on line. 2016; 10 (12):4432-42.Anjos M, Oselame G (2013). Nursing Care for Elderly Patients with Arteriovenous Fistula in Hemodialysis Therapy. Rev. Uniand. 2013; 14(3): 251-62.

- Hafezieh A., Dehghan M., Taebi M., \& Iranmanesh S (2020): Self-management, selfefficacy and knowledge among patients under haemodialysis: a case in Iran. Journal of Research in Nursing, 2020: 25 (2). 128 - 138.

- Ikiz S, Usta Y, Sousa C, Teles P, Dias V, Magalhães A, , \& Ribeiro O, (2020): Validation of the scale of assessment of self-care behaviours for arteriovenous fistula in patients ongoing haemodialysis in Turkey $\mathrm{J}$ Ren Care; 2020; 2020;10.1111/jorc. 12354 .

- Jager K, Kovesdy C., Langham R., Rosenberg M, Jha V, \& Zoccali C. (2019): A single number for advocacy and communication-worldwide more than 850 million individuals have kidney diseases. Nephrology, dialysis, transplantation : official publication of the European Dialysis and Transplant Association - European Renal Association, 2019: 34(11), 1803-1805.

- Jonkman N, Groenwold R, Trappenburg, A, \& Schuurmans M (2017): Complex self-management interventions in chronic disease unravelled: a review of lessons learned from an individual patient data meta-analysis. J Clin Epidemiol. 2017 Mar; 83:48-56.

- Kim B, \& Kim J (2019): Influence of Uncertainty, Depression, and Social Support On Self-Care Compliance In Hemodialysis Patients. Ther Clin Risk Manag. 2019;15:1243-1251 
- Lok C, Huber T, Lee T, Shenoy S, Yevzlin A, Abreo K, \& Allon M, (2020): KDOQI Clinical Practice Guideline for Vascular Access: 2019 Update. Am J Kidney Dis. 2020; 75 (Suppl 2):S1S164.

- Mahjubian A, Bahraminejad N, \& Kamali K (2018): The Effects of Group Discussion Based Education on the Promotion of Self-Management Behaviors in Hemodialysis Patients. J Caring Sci. 2018; 7(4):225-232.

- Mahmoud S, Mohga S, \& Abdel Raouf H (2014): Assessment of Self-Care Practice of Patients on Maintenance Hemodialysis at Cairo University Hospitals. Journal of Education and Practice. 2014: (5) 39: 161-147.

- Ministry of Health - Democratic Republic of the Congo (2014). Clinical guidelines for the care of patients with kidney disease, 2014. Available at : http://bvsms.saude.gov.br/bvs/publicacoes/diretrizes clinicas cuidado_paciente renal.pdf.

- Mosavi F, Aliakbari F, \& Rabiei L (2020). Effect of education based on "PRECEDE" model on selfcare behavior in hemodialysis patients. J Educ Health Promot. 2020; 31; 9:69.

- Ozen, N, Tuson N, \& Cinar F (2017): Investigation of the knowledge and attitude of patients who are undergoing hemodialysis treatment regarding their arteriovenous fistula. J Vasc access 2017; 18 (1):64-68.

- Parvan K, Hasankhani H, Seyyedrasooli A, Riahi S, \& Ghorbani M (2015): The effect of two educational methods on knowledge and adherence to treatment in hemodialysis patients: clinical trial. J Caring Sci. 2015; 4(1):83-93.

- Pretto C, Winkelmann E, Hildebrandt L, Barbosa D , \& Stumm F (2020): Quality of life of chronic kidney patients on hemodialysis and related factors. Rev. Latino-Am. Enfermagem 2020; 28:e3327.

- Qian Q (2017): Electrolyte and acid--base disorders in chronic kidney disease and end-stage kidney failure. Blood Purif. 2017; 43(1-3): 179-88

- Rachmadi A \&Ratnasari I, Nursalam A, \& Wibowo A (2020): Relationship between Self-Care for Fluid Limitation and Interdialytic Weight Gain among Patients with Hemodialysis at RatuZalecha Hospital, Martapura. Indian Journal of Public Health Research \& Development.2020; 1(11): 995.

- Rahimi F, Oskouie F, Naser O, Sanandj M, \& Gharib, A (2017): The effect of self-care on patients undergoing Hemodialysis in the Sanandaj Hospitals affiliated to Kurdistan University of Medical Sciences in 2016. Bali Medical Journal. 2017; 6(3): 684-689.
- Rainey H(2018): Managing chronic kidney disease in primary care, Nurse Prescribing $2018 ; 16$ (11)542-48

- Ramezani T, Gharlipour Z., Sharifirad, G, \& Mohebi S. (2019): Effect of the Education Based on the Self-Efficacy Theory on Skin and Fistula Care in Hemodialysis Patients. Journal Of Health And Care, 20(4), 342-353. doi: 10.29252/jhc.20.4.342

- Ramezani T, Sharifirad G, Rajati F, Rajati M, Mohebi S. Effect of educational intervention on promoting self-care in hemodialysis patients (2019): Applying the self-efficacy theory (2019). J Educ Health Promot.2019; 14; 8:65.

- Rashid N., Aamer M., Malik U., Arif N, Akram M \& Irshad A (2018): Arteriovenous fistula (AVF) self-care: A study at a Tertiary Care Hospital in Lahore, Pakistan. Annals of King Edward Medical University. 2018; 24(1): 134-140.

- Riegel B, Westland H, Iovino P, Barelds I, Bruins S, \& Strömberg A, (2021): Characteristics of self-care interventions for patients with a chronic condition: A scoping review. Int J Nurs Stud. 2021; 116:103713.

- Rini S., Rahmayani, T, Sari, E, \& Lestari R. (2021): Differences in the quality of life of chronic kidney disease patients undergoing hemodialysis and continuous ambulatory peritoneal dialysis. Journal of Public Health Research, 10(2):2209.

- Santana M, Silva D, Echevarría-Guanilo M, Lopes S, Romanoski P, \& Böell J (2020): Selfcare in individuals with chronic kidney disease on hemodialysis. Rev GauchaEnferm. 2020 ;(5);41:e20190220

- Soliman AR, Fathy A, \& Roshd D (2012): The growing burden of end-stage renal disease in Egypt. E pub., 2012; 34(4):425-8.

- Tsai Y, Wang S, Tsai H, Chen T, Kung L, Hsiao P, \& Hsiao S (2021): The interaction between selfcare behavior and disease knowledge on the decline in renal function in chronic kidney disease. Sci Rep. 2021; 11;11(1):401

- Wang S, Chiu Y, Kung L, Chen T, Hsiao S, Hsiao P, Hwang S, \& Hsieh H (2019): Patient assessment of chronic kidney disease self-care using the chronic kidney disease self-care scale in Taiwan. Nephrology (Carlton). 2019 24(6):615-621.

- Webster AC, Nagler E, Morton RL, \& Masson P. (2017): Chronic Kidney Disease. Lancet. 2017 Mar 25; 389 (10075):1238-52.

- Westland H, Ågren S, Atienza F, Blue L, Heisler M, \& Jaarsma T, (2016): Do Self-Management Interventions Work in Patients With Heart Failure? An Individual Patient Data Meta-Analysis. Circulation. 2016 Mar 22; 133(12):1189-98. 
- Whdan S, Soliman N, Mekhemar S, \& Abd ElMoneem H (2019): Home self-care for client with hemodialysis at Ashmon district Hospital.Egyptian Journal of Health Care, 2019; 10 (3)

- Wong K, Velasquez A, Powe N, \& Tuot D (2018): Association between health literacy and self-care behaviors among patients with chronic kidney disease. BMC Nephrol. 2018;19(1):196.

- Xie, Y., Bowe, B., Mokdad, A., Xian, H., Yan, Y., Li, T., Maddukuri, G., Tsai, C., Floyd, T., \& AlAly, Z. (2018): Analysis of the Global Burden of Disease study highlights the global, regional, and national trends of chronic kidney disease epidemiology from 1990 to 2016. Kidney international, 94(3), 567-581.

- Yang M, Zhao H, Ding X, Zhu G, Yang Z, Ding L, Yang X, Zhao Y, Chen L, Yang R, Fang J, \& Sousa C (2019): Self-Care Behavior of Hemodialysis Patients With Arteriovenous Fistula in China: A Multicenter, Cross-Sectional Study. TherApher Dial. 2019 Apr; 23 (2):167-172.

- ZainELdin, N Omar T, Younis J, \& Ahmed G (2018): Effect of Self-Care Model Intervention on Quality of Life of Children Undergoing Hemodialysis. Res \& Rev Health Care Open Acc J .2018: 2(2)129-137. 